\title{
Thalidomide, A Rational Agent for Treatment of Multicentric Reticulohistiocytosis
}

Daniel J Zinn ${ }^{1}$, Olive Eckstein ${ }^{2}$, Mary L Olsen ${ }^{3}$, Carl E Allen ${ }^{2}$ and Kenneth L McClain²*

${ }^{1}$ Department of Pediatrics, Lehigh Valley Reilley Children's Hospital, Allentown, Pennsylvania, USA

${ }^{2}$ Texas Children's Cancer and Hematology Centers, Baylor College of Medicine, Houston, Texas, USA

${ }^{3}$ Baptist Physicians Network, Rheumatology Center, Beaumont, Texas, USA

"Corresponding author: Kenneth L McClain, Texas Children's Cancer and Hematology Centers, Baylor College of Medicine, Houston, Texas, USA, Tel: 8328224208; Fax: 8328251503; E-mail: 77030 klmcclai@txch.org

Received date: March 14, 2019; Accepted date: March 29, 2019; Published date: April 08, 2019

Copyright: ( 2019 Zinn DJ, et al. This is an open-access article distributed under the terms of the Creative Commons Attribution License, which permits unrestricted use, distribution, and reproduction in any medium, provided the original author and source are credited.

\begin{abstract}
A patient with Multifocal Reticulohistiocytosis (MRH) of skin and joints failed treatment with etanercept, methotrexate, hydroxychloroquine, prednisone, bisphosphonates and hydroxyzine. Long term treatment with thalidomide led to marked improvement in joint and cutaneous manifestations.
\end{abstract}

Keywords: Reticulohistiocytosis; Multicentric reticulohistiocytosis; Histiocytic disorders; Thalidomide

\section{Introduction}

Multicentric Reticulohistiocytosis (MRH) is a rare non-Langerhans Cell Histiocytosis affecting skin and joints first described in 1937 by Webber and Freudenthal [1]. The incidence of MRH is not known and most often presents in 40-50-year-old women [2,3]. Skin lesions are red, brown or violaceous papules and nodules on the hands, fingers, face, and trunk ranging in size from several millimeters to several centimeters and can be associated with pruritus [3]. Clusters of periungal papules, often referred to as 'coral beads', are considered pathognomonic of MRH. Arthritis associated with MRH frequently involves the distal and proximal interphalangeal joints, but may also involve other joints. The destructive process associated with this disease can progress to arthritis mutilans, which has been reported in up to $50 \%$ of cases if untreated [3]. While less common, systemic symptoms can include fever, weight loss, anorexia, weakness or lymphadenopathy $[2,3]$. Twenty-five to $50 \%$ of MRH cases have been associated with a variety of malignancies such as breast, ovarian, stomach and cervical cancers, lymphoma, and melanoma as well as autoimmune disorders in 5\%-20\% of cases, most commonly Sjögrens syndrome [4-6].

\section{Case Report}

At the age of 36-years, this African-American woman developed fatigue, decreased appetite, joint pain, Raynaud's phenomenon, alopecia, oral ulcers, sicca syndrome, 50-pound weight loss in one month, and progressive weakness resulting difficulty in getting out of bed or sitting without assistance. Laboratory evaluation demonstrated abnormal levels of Antinuclear Antibodies (ANA) with specific elevations of anti-RNP and anti-Ro/SSA antibodies and she was diagnosed with systemic lupus erythematous/Sjögrens syndrome. Treatment with oral steroids was initiated which provided partial relief of some symptoms, but her strength and mobility did not normalize for several weeks. Approximately six months later, she developed nodular cutaneous lesions on her fingers, arms, and chest and back which were associated with intense pruritus. Biopsy of a nodule showed pathologic features consistent with MRH. In spite of combination treatment with methotrexate, etanercept, hydroxychloroquine, prednisone, bisphosphonates and hydroxyzine, her reticulohistiocytosis persisted. She was referred to our adult clinic at age 40 years. Her exam was notable for a crusted papular rash on her scalp, diffuse nodules, and papules in the periorbital region, and numerous cutaneous lesions deforming her hands and fingers (Figure 1). Treatment with thalidomide $100 \mathrm{mg}$ daily was started and after three months she had partial improvement of her disease with decreased size of her nodules and no new sites. After seven months of this combination therapy, etanercept was successfully discontinued. Approximately five years later, tocilizumab was added to manage symptoms of arthritis. After 9 years her lesions were nearly resolved, but thalidomide was temporarily unavailable for one month and her skin papules grew in size and number, most prominently below the left eye. Thalidomide was restarted and the papules subsequently improved. She has remained on thalidomide for over 12.5 years, with doses ranging from 100 to $350 \mathrm{mg}$ daily. Reduction of the thalidomide dose below $200 \mathrm{mg}$ daily resulted in intense pruritus at sites where lesions had previously existed.

In January 2018 she was switched to lenalidomide because of increasing insomnia. The patient currently receives lenalidomide 25 $\mathrm{mg}$ daily, in combination with hydroxychloroquine, methotrexate, and tocilizumab, with an overall excellent response and minimal toxicities limited to mild insomnia, peripheral neuropathy in the lower extremities which she finds tolerable, and mild leukopenia which resolved after dose reduction.

At her most recent clinical assessment, her digital and periorbital lesions had almost completely resolved (Figure 2) although she has persistent scalp nodules and ongoing chronic pain in the hands, knees, and ankles. 


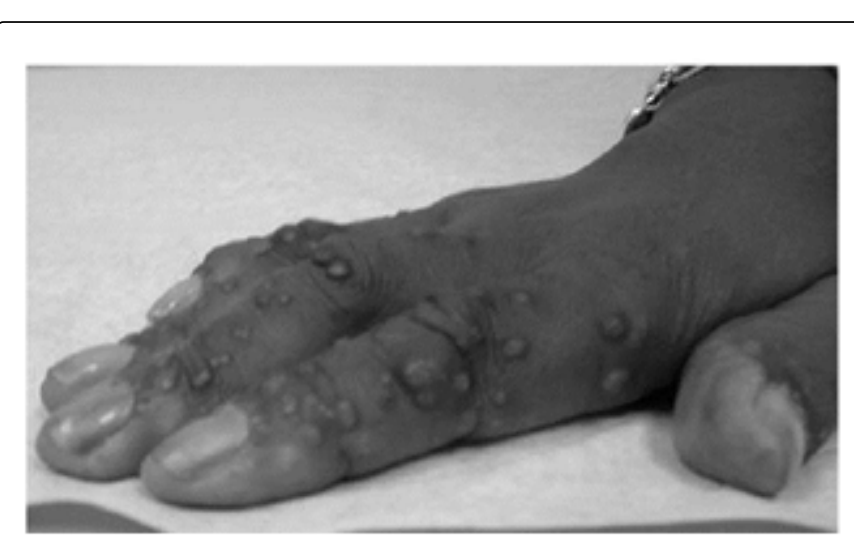

Figure 1: The patient presented with numerous skin lesions deforming her hands, including clusters of periungal papules, often referred to as 'coral beads.'

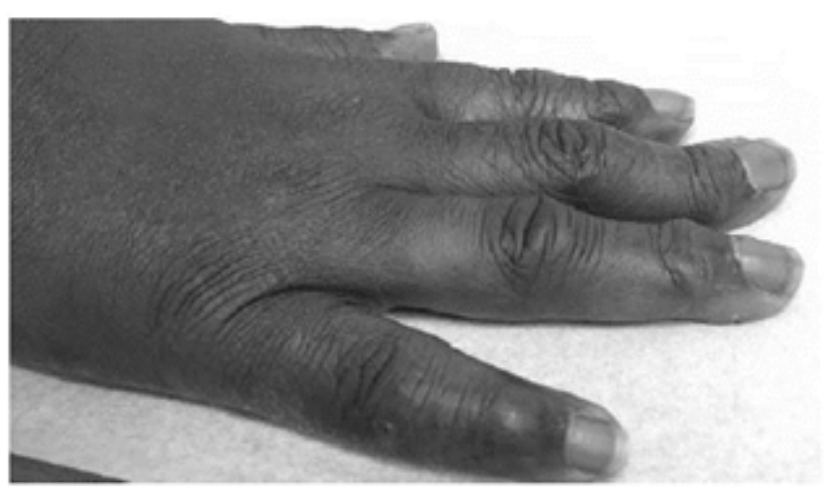

Figure 2: After more than 10 years of therapy with thalidomide the patient had a resolution of the skin lesions on her hands. Destruction of her joints due to inflammatory arthritis is demonstrated in the distal interphalangeal joints.

\section{Discussion}

Given the possibilities of an underlying malignancy or progressive arthritis, early diagnosis along with thorough cancer screening and prompt initiation of treatment is critical in MRH. The diagnosis is typically made with a biopsy and histology shows large multinucleated giant cells with eosinophilic glassy cytoplasm, positive staining for Periodic Acid Schiff (PAS), and CD-68, but negative for S100, CD1a, and factor XIIIa [7]. The pathogenesis of MRH is poorly understood, although immunohistochemical markers suggest that the pathologic cells of MRH are derived from a monocyte/macrophage lineage [7]. Synovial fluid from patients with MCR shows numerous macrophages and elevated levels of Tumor Necrosis Factor alpha (TNF- $\propto$ ), interleukin-6 (IL-6), and interleukin-1 beta (IL-1 $\beta$ ) resulting in the osteoclastic activity of the pathologic cells and leading to destructive arthritis $[8,9]$.

There is no definitive standard of care for patients with MRH. Given the high risk of progressive joint destruction and potentially disfiguring skin lesions, effective and timely therapy is crucial to patient outcomes. Past treatment strategies have utilized immunosuppressive or cytotoxic therapies including corticosteroids, hydroxychloroquine, cyclophosphamide, chlorambucil, methotrexate, and nonsteroidal anti-inflammatory drugs. More recently, biological agents which target TNF- $\propto$, including etanercept, adalimumab, and infliximab, have induced partial or complete clinical responses $[3,10,11]$. Bisphosphosphanates have also been utilized with some success based on a proposed mechanism of action in which these drugs inhibit histiocytic differentiation and osteoclastic activity $[3,4,11]$.

Thalidomide has previously been reported as an ineffective treatment in a patient with MRH who had concurrent hepatic carcinoma. However, after treatment directed at the malignancy, the patient had clinical improvement of their MRH [12]. Our patient had no evidence of a concurrent neoplasm. Her skin lesions gradually improved with thalidomide treatment but progressed when she stopped the medication for a brief period of time. Thalidomide and lenalidomide, a thalidomide derivative, have been effective in the treatment of Langerhans Cell Histiocytosis (LCH) and non-LCH histiocytic diseases [13,14]. These agents are classified as immunomodulatory drugs and have been shown to have an antiinflammatory action by modulating cytokine production and release [15].

\section{Conclusion}

Thalidomide has been shown to decrease the production of TNF- $\alpha$, IL-6, and IL-1, making it a therapeutically rational choice in the treatment of MRH. MRH is a rare disease that can result in disfiguring and painful lesions of the skin and joints. It may be associated with malignancy, and a full age-appropriate cancer screening must be completed. If an underlying malignancy is not identified, thalidomide, or its related derivative lenalidomide, should be considered as adjunctive agents. Thalidomide is a rational agent that is generally well tolerated, and can be used in combination with other biologic treatments directed at MRH.

\section{Acknowledgment}

This work was performed with support from the HistioCure foundation (Texas Children's Cancer Center Histiocytosis Program). Additional grant support includes NIH R01 (CA154489), and Dan L. Duncan Cancer Center support grant (P30CA1215123).

\section{Ethics Approval and Consent to Participate}

This case review was conducted according to the guidelines for use of human subjects in research of the Baylor College of Medicine Institutional Review Board.

\section{Patient Consent for Publication}

The patient signed a consent allowing publication of her clinical data.

\section{Conflict of Interest Disclosures}

Mary L. Olsen is on the Speakers Bureau for Amgen, the maker of Enbrel (etanercept.) All other authors have no conflicts of interest to disclose. 


\section{References}

1. Weber FP, Freudenthal W (1937) Nodular Non-diabetic Cutaneous Xanthomatosis with Hypercholesterolaemia and Atypical Histological Features. Proc R Soc Med 30: 522-526.

2. Luz FB, Gaspar TAP, Kalil-Gaspar N, Ramos-e-Silva M (2001) Multicentric reticulohistiocytosis. J Eur Acad Dermatol Venereol 15: 524-531.

3. Tariq S, Hugenberg ST, Hirano-Ali SA, Tariq H (2016) Multicentric reticulohistiocytosis (MRH): Case report with review of literature between 1991 and 2014 with in depth analysis of various treatment regimens and outcomes. SpringerPlus 5: 180 .

4. Catterall MD, White JE (1978) Multicentric reticulohistiocytosis and malignant disease. Br J Dermatol 98: 221-224.

5. Snow JL, Muller SA (1995) Malignancy-associated multicentric reticulohistiocytosis: A clinical, histological and immunophenotypic study. Br J Dermatol 133: 71-76.

6. Ben AK, Mahmoud I, Chatelus E, Sordet C, Gottenberg JE, et al. (2010) Multicentric reticulohistiocytosis: An autoimmune systemic disease? Case report of an association with erosive rheumatoid arthritis and systemic Sjogren syndrome. Joint Bone Spine 77: 274-276.

7. Gorman JD, Danning C, Schumacher HR, Klippel JH, Davis JC (2000) Multicentric reticulohistiocytosis: Case report with immunohistochemical analysis and literature review. Arthritis Rheum 43: 930-938.
8. Adamopoulos IE, Wordsworth PB, Edwards JR, Ferguson DJ, Athanasou NA (2006) Osteoclast differentiation and bone resorption in multicentric reticulohistiocytosis. Hum Pathol 37: 1176-1185.

9. Bennassar A, Mas A, Guilabert A, Julia M, Mascaro-Galy JM, et al. (2011) Multicentric reticulohistiocytosis with elevated cytokine serum levels. J Dermatol 38: 905-910.

10. Zhao H, Wu C, Wu M, Zhou Y, Zhu H, et al. (2016) Tumor necrosis factor antagonists in the treatment of multicentric reticulohistiocytosis: Current clincal evidence. Mol Med Rep 14: 209-217.

11. Macia-Villa CC, Zea-Mendoza A (2016) Multicentric reticulohistiocytosis: Case report with response to infliximab and review of treatment options. Clin Rheumatol 35: 527-534.

12. Han L, Huang Q, Liao KH (2012) Multicentric reticulohistiocytosis associated with liver carcinoma: Report of a case. Case Rep Dermatol 4: 163-169.

13. McClain KL, Kozinetz CA (2007) A phase II trial using thalidomide for Langerhans cell histiocytosis. Pediatr Blood Cancer 48: 44-49.

14. Rubinstein M, Assal A, Scherba M (2016) Lenalidomide in the treatment of Rosai Dorfman disease-A first in use report. Am J Hematol 91(2): E1.

15. Anderson KC (2005) Lenalidomide and thalidomide: Mechanisms of action-similarities and differences. Semin Hematol 42: S3-8. 